\title{
Commentary: Health-Related Quality of Life and Burden of Illness in Adults with Newly Diagnosed Attention-Deficit/Hyperactivity Disorder in Sweden
}

\author{
E. Ahnemark ${ }^{1 *}$, M. Di Schiena ${ }^{2}$, A.-C. Fredman²,8, E. Medinn ${ }^{3,4}$, J. K. Söderling ${ }^{5}$, Y. Ginsberg ${ }^{6,7}$ \\ 'Shire, Vasagatan 7, SE-11120 Stockholm, Sweden \\ ${ }^{2}$ Prima Child and Adult Psychiatry AB, Stockholm, Sweden \\ 3PAREXEL International, Stockholm, Sweden \\ ${ }^{4}$ Department of Learning, Informatics, Management and Ethics, Karolinska Institute, Stockholm, Sweden \\ ${ }^{5}$ Bell Analytics, Stockholm, Sweden \\ ${ }^{6}$ Department of Medical Epidemiology and Biostatistics, Karolinska Institute, Stockholm, Sweden \\ ${ }^{7}$ Department of Clinical Neuroscience, Centre for Psychiatry Research, Karolinska Institute, Stockholm, Sweden \\ ${ }^{8}$ Psychiatry Centre, Stockholm County Council, Södertälje, Sweden
}

Article Info

\section{Article Notes}

Received: September 10, 2018

Accepted: October 18, 2018

\section{*Correspondence:}

Dr. Ewa Ahnemark, MD, Shire, Vasagatan 7, SE-111 20

Stockholm, Sweden; Telephone No: +46.8.54496443; Email: eahnemark@shire.com.

(c) 2018 Ahnemark E This article is distributed under the terms of the Creative Commons Attribution 4.0 International License.

\section{Background and Study Overview}

Often diagnosed during childhood, attention-deficit/ hyperactivity disorder (ADHD) is now recognized to persist into adulthood in approximately $65 \%$ of cases ${ }^{1-4}$. Recent evidence has also proposed that an adult-onset form of ADHD may exist in some populations ${ }^{5,6}$. ADHD can have a significant negative impact on professional, financial, social and emotional aspects of adult life $\mathrm{e}^{7,8}$ and our first report of this study published elsewhere ${ }^{9}$ adds to the limited published information related to the burden of ADHD in a population of adults (Swedish) with newly diagnosed ADHD.

In this commentary on our original report ${ }^{9}$, we describe the health-related quality of life (HRQoL) evaluated in an adult study population in Sweden and highlight two specific demographic factors that were found to impact HRQoL, namely gender and the presence of comorbid psychiatric disorders.

The observational, cross-sectional, retrospective chart review enrolled 189 participants, of mean age 35 years, with newly diagnosed ADHD from two specialist outpatient clinics in Stockholm, Sweden. The study enrolled participants who presented with ADHD symptoms from a young age but remained undiagnosed until adulthood, despite the persistence of symptoms. Data extracted from patient records (2013-2015) included demographics, clinical characteristics and comorbid psychiatric diagnoses. HRQoL was assessed at diagnosis, and evaluated using the self-rated fivedimension EuroQol questionnaire (EQ-5D). Linear regression was used to identify factors that may influence HRQoL.

\section{HRQoL in Adults with ADHD was Poor}

Using the EQ-5D instrument, in which a score of 1.0 represents perfect health and 0 represents death, the present population of adults with ADHD were found to have a mean index score of 0.63 . For context, this value is lower than any of those published for the healthy adult population in Sweden, which range from $0.74-0.89^{10}$. Furthermore, because the EQ-5D is a generic instrument, HRQoL can be compared across diseases and the mean score observed in this adult ADHD population was similar to or lower than those reported 
for groups of patients with other chronic conditions such as heart failure (0.64) and diabetes $(0.72)^{11}$.

\section{Psychiatric and Somatic Comorbidities Predicted Poor HRQOL}

ADHD often co-occurs with other psychiatric disorders in adults ${ }^{12,13}$. Based on the Mini International Neuropsychiatric Interview (MINI), almost half of participants (49\%) in the present study were found to have at least one psychiatric comorbidity, with anxiety and depression the most common. The presence of comorbid anxiety and depression was significantly associated with poor HRQoL, and the presence of both anxiety and depression together predicted even poorer HRQoL than either comorbidity alone. Furthermore, multiple psychiatric comorbidities predicted poor HRQoL even when individual MINI diagnoses alone did not predict poor HRQoL. The severity of the symptoms of depression was assessed using the self-reported Montgomery-Åsberg Depression Rating Scale (MADRS-S), and the mean score of 19.8 in the overall study population is indicative of mildto-moderate levels of depression. Indeed, a high MADRS-S score (indicative of more severe symptoms of depression) was the strongest predictor of poor HRQoL.

Co-occurrence of ADHD with other psychiatric disorders can impede the diagnosis of ADHD. A recent registry study found that adults with ADHD symptoms may undergo years of psychiatric treatment before receiving an ADHD diagnosis, with patients waiting, on average, 3 years from the initial point of contact with psychiatric services ${ }^{14}$. These findings emphasize the importance of considering whether ADHD is a factor in adults that are referred to psychiatric clinics with multiple symptoms.

We also observed that the majority of participants in the present study (77\%) had at least one somatic comorbidity, supporting previous reports that adults with ADHD are more likely to have somatic disorders than those without ${ }^{15}$. Like psychiatric comorbidities, somatic comorbidities may also complicate the diagnosis of ADHD. For example, reduced physical activity due to asthma or joint problems may mask the symptoms of hyperactivity, whereas the effect of chronic pain on the ability to concentrate may resemble the symptoms of inattention associated with ADHD. In addition, symptoms of thyroid disease including tiredness, poor memory and concentration, and slow movements, thought and speech may also resemble $\mathrm{ADHD}^{16,17}$. Thus, interpreting the expression of symptoms in the context of an individual's wider medical history is paramount for the accurate diagnosis of ADHD and comorbidities.

\section{Female Gender Predicted Poor HRQoL}

The prevalence of ADHD is reportedly higher in males than females ${ }^{18}$. However, because the externalizing symptoms of hyperactivity and impulsivity typically associated with males with ADHD are more likely to result in referral and diagnosis than the symptoms of inattention more commonly associated with females, it may be speculated that ADHD in females is under-recognized. Thus, the preponderance of females $(57 \%$ of the study population were female) in our study is uncommon in the published literature and provides an unusual opportunity to gain a valuable insight into the impact of ADHD in adults generally and females in particular. In our article, we reported that HRQOL was statistically significantly poorer in females with ADHD than in males.

The adjusted mean EQ-5D total score was 0.08 points lower in females than males, although the difference only just reached statistical significance $(p=0.049)^{9}$. According to a 2001 report, the mean EQ-5D total score is also lower in females than in males in the general Swedish population (by 0.03 points, $p<0.001)^{10}$, a difference which may have been reflected in our study. Co-morbid anxiety and depression are reportedly more common in adult females than males with $\mathrm{ADHD}^{19,20}$, and a preponderance of comorbid anxiety or depression in the female patients in our study might account for the lower EQ-5D scores, but further analysis is required. An interaction between psychiatric co-morbidities and gender in adults with ADHD has been proposed $^{20}$ however another study reported no effect of gender on the association between ADHD and other psychiatric disorders ${ }^{13}$. Rates of suicidal ideation are also significantly higher in females with ADHD than in those without. Suicidal ideation in females was significantly associated with 'problems with self-concept" ${ }^{\prime 21}$, which highlights the need for careful assessment of internalized symptoms that are often difficult to recognize, especially in females. Further studies evaluating the interaction between gender and co-morbidities in adults with ADHD are warranted.

\section{Implications for the Clinical Management of Patients with ADHD}

The findings of our study have several implications for clinical practice. First, the presence of somatic and psychiatric comorbidities may influence an individual's ability to follow instructions and adhere to medication, and the level of functional impairment should be considered when selecting treatment. Second, patients with multiple comorbidities may be more prone to poor HRQoL and, although emerging evidence suggests that ADHD treatments may have a positive impact on HRQoL ${ }^{22}$, therapeutic strategies should aim to address poor HRQoL as well as symptoms. Finally, gender differences may occur in the presentation of ADHD and comorbid disorders, underpinning the need for individualized, evidence-based treatment strategies. 


\section{Conclusions}

The findings described in our paper demonstrate that the burden of ADHD in adults with the disorder includes poor HRQoL. That poor HRQoL was associated with comorbidities and female gender highlights the importance of considering the possibility that ADHD may be a factor when adults of either gender present with psychiatric symptoms. There is a need for more research in this field.

\section{References}

1. Biederman J. Advances in the neurobiology of ADHD. CNS Spectr. 2007; 12(4 Suppl 6): 6-7.

2. Faraone SV, Biederman J, Mick E. The age-dependent decline of attention deficit hyperactivity disorder: a meta-analysis of follow-up studies. Psychol Med. 2006; 36(2): 159-65.

3. Nylander L, Holmqvist M, Gustafson L, et al. ADHD in adult psychiatry. Minimum rates and clinical presentation in general psychiatry outpatients. Nord J Psychiatry. 2009; 63(1): 64-71.

4. Ginsberg Y, Hirvikoski T, Lindefors N. Attention deficit hyperactivity disorder (ADHD) among longer-term prison inmates is a prevalent, persistent and disabling disorder. BMC Psychiatry. 2010; 10(112).

5. Agnew-Blais JC, Polanczyk GV, Danese A, et al. Evaluation of the persistence, remission, and emergence of attention-deficit/ hyperactivity disorder in young adulthood. JAMA Psychiatry. 2016; 73(7): 713-20.

6. Caye A, Rocha T, Anselmi L, et al. Attention-deficit/hyperactivity disorder trajectories from childhood to young adulthood: Evidence from a birth cohort supporting a late-onset syndrome. JAMA Psychiatry. 2016; 73(7): 705-12.

7. Brod M, Pohlman B, Lasser R, et al. Comparison of the burden of illness for adults with ADHD across seven countries: a qualitative study. Health Qual Life Outcomes. 2012; 10(47).

8. Brod M, Schmitt E, Goodwin M, et al. ADHD burden of illness in older adults: a life course perspective. Qual Life Res. 2012; 21(5): 795-9.

9. Ahnemark E, Di Schiena M, Fredman AC, et al. Health-related quality of life and burden of illness in adults with newly diagnosed attentiondeficit/hyperactivity disorder in Sweden. BMC Psychiatry. 2018; 18(1): 223.

10. Burstrom K, Johannesson M, Diderichsen F. Swedish population health-related quality of life results using the EQ-5D. Qual Life Res. 2001; 10(7): 621-35.

11. Peters M, Crocker H, Jenkinson C, et al. The routine collection of patient-reported outcome measures (PROMs) for long-term conditions in primary care: a cohort survey. BMJ Open. 2014; 4(2): e003968.

12. Sobanski E. Psychiatric comorbidity in adults with attention-deficit/ hyperactivity disorder (ADHD). Eur Arch Psychiatry Clin Neurosci. 2006; 256 Suppl 1: i26-31.

13. Biederman J, Faraone SV, Monuteaux MC, et al. Gender effects on attention-deficit/hyperactivity disorder in adults, revisited. Biol Psychiatry. 2004; 55(7): 692-700.

14. Nylander L, Holmqvist M, Gustafson L, et al. Attention-deficit/ hyperactivity disorder (ADHD) and autism spectrum disorder (ASD) in adult psychiatry. A 20 -year register study. Nord J Psychiatry. 2013; 67(5): 344-50.

15. Hodgkins P, Montejano L, Sasané R, et al. Cost of illness and comorbidities in adults diagnosed with attention-deficit/hyperactivity disorder: a retrospective analysis. Prim Care Companion CNS Disord. 2011; 13(2).

16. Samuels MH. Psychiatric and cognitive manifestations of hypothyroidism Curr Opin Endocrinol Diabetes Obes. 2014; 21(5): 377-83.

17. Garber JR, Cobin RH, Gharib H, et al. Clinical practice guidelines for hypothyroidism in adults: cosponsored by the American Association of Clinical Endocrinologists and the American Thyroid Association. Thyroid. 2012; 22(12): 1200-35.

18. Willcutt EG. The prevalence of DSM-IV attention-deficit/hyperactivity disorder: a meta-analytic review. Neurotherapeutics. 2012; 9(3): 490-9.

19. Wilens TE, Biederman J, Faraone SV, et al. Presenting ADHD Symptoms, Subtypes, and Comorbid Disorders in Clinically Referred Adults with ADHD. J Clin Psychiatry. 2009; 70(11): 1557-62.

20. Solberg BS, Halmoy A, Engeland A, et al. Gender differences in psychiatric comorbidity: a population-based study of 40000 adults with attention deficit hyperactivity disorder. Acta Psychiatr Scand. 2018; 137(3): 176-86.

21. Kakuszi B, Bitter I, Czobor P. Suicidal ideation in adult ADHD: Gender difference with a specific psychopathological profile. Compr Psychiatry. 2018; 85: 23-9.

22. Coghill D. The impact of medications on quality of life in attentiondeficit hyperactivity disorder: a systematic review. CNS Drugs. 2010; 24(10): 843-66. 